\title{
Silencing of CD86 in dendritic cells by small interfering RNA regulates cytokine production in $T$ cells from patients with allergic rhinitis in vitro
}

\author{
RONG SUN $^{1}$, YANG YANG $^{2}$, ZHENG GU $^{2}$, XINYE TANG $^{2}, \mathrm{CHENG} \mathrm{ZHANG}^{2}$, WEI KOU $^{2}$ and PING WEI ${ }^{2}$ \\ ${ }^{1}$ Department of Physical Examination, The First Affiliated Hospital of Chongqing Medical University; ${ }^{2}$ Department of \\ Otorhinolaryngology, The Children's Hospital of Chongqing Medical University, Chongqing 400016, P.R. China
}

Received May 20, 2018; Accepted July 30, 2019

DOI: $10.3892 / \mathrm{mmr} .2019 .10638$

\begin{abstract}
The aim of the present study was to investigate the expression and role of the co-stimulatory molecule T-lymphocyte activation antigen CD86 (CD86) in dendritic cells (DCs) from the peripheral blood of patients with allergic rhinitis (AR) compared with those from healthy individuals. It was observed that mature DCs from the peripheral blood of patients with AR expressed high levels of the co-stimulatory molecule CD86, but not CD80, compared with healthy control subjects. CD86 expression levels in DCs decreased significantly following transfection with siRNA in a lentiviral vector. Furthermore, the level of transforming growth factor- $\beta 1$ produced by $\mathrm{T}$ cells co-cultured with DCs was significantly increased in the siRNA group, while interleukin (IL)-4 and IL-5 production was significantly decreased. The findings of the present study indicated that CD86 may play a pivotal role in the regulatory $\mathrm{T}$ cell/type 2 helper $\mathrm{T}$ cell imbalance in allergic inflammation.
\end{abstract}

\section{Introduction}

Allergic rhinitis (AR) is a chronic nasal mucosal inflammation characterized by infiltration and activation of a number of immune cells $(1,2)$. Type 2 helper T (Th2) cells and regulatory $\mathrm{T}$ (Treg) cells play an important role in AR $(3,4)$. A deficiency of Treg cells and higher numbers of Th2 cells are the key factors underlying the development of allergic inflammation of the nasal mucosa (5). T cells can regulate immune function by secreting cytokines and proinflammatory molecules.

Correspondence to: Professor Xinye Tang, Department of Otorhinolaryngology, The Children's Hospital of Chongqing Medical University, 136 Zhongshan 2nd Road, Yuzhong, Chongqing 400016, P.R. China

E-mail: ent2002@163.com

Key words: allergic rhinitis, T-lymphocyte activation antigen CD86, small interfering RNA, dendritic cells, regulatory T cells
Transforming growth factor (TGF)- $\beta$, interleukin (IL)-5 and IL-4 are typical cytokines secreted by Treg and Th2 cells.

Dendritic cells (DCs) are an important type of antigen-presenting cell that regulate the activation and differentiation of T cells. Antigen-activated DCs display co-stimulatory molecules, which determine whether naïve $\mathrm{T}$ cells differentiate into type 1 helper $\mathrm{T}$ (Th1), Th2 or Treg cells $(6,7)$. A previous study demonstrated that mature DCs (mDCs) express high levels of the co-stimulatory molecules T-lymphocyte activation antigen CD80 (CD80) and CD86, which provide the signals that trigger the activation, proliferation and differentiation of T cells by interacting with CD28 (8). Despite sharing the same $\mathrm{T}$ cell stimulatory receptor, CD80 and CD86 induce different DC:T cell interactions through alternative pathways. CD80 may be more potent in inducing an antitumor immune response compared with CD86, while CD86 preferentially induces Th2-driven allergic responses $(9,10)$. Vermaelen and Pauwels (11) demonstrated that airway DCs in a mouse model of asthma expressed a high level of CD86, but not CD80. Consistent with this, an allergen challenge with ovalbumin (OVA) in a murine model of airway inflammation led to the maturation of lung DCs and increased expression of CD86 (12). However, it is unclear if the upregulation of CD86 in DCs may play a critical role in the development of AR.

DCs have been shown to be important in AR. The number of DCs in the nasal mucosa of patients with AR were dramatically reduced following intranasal corticosteroid therapy $(13,14)$. Moreover, KleinJan et al (15) reported that DCs in the nasal mucosa of symptomatic AR patients displayed a more mature phenotype (expressing CD86). In previous studies on AR models, mDCs have been shown to upregulate the expression of the Th2-cell cytokines IL-4, IL-5 and IL-13, and induce eosinophilic inflammation $(15,16)$. However, the effects of the knockdown of CD86 in DCs on the differentiation and cytokine secretion by T cells in AR are not yet known.

RNA interference is an approach to gene silencing. Small interfering RNAs (siRNAs) are small non-coding RNA molecules that are complementary to the mRNA transcript of the target gene. These small RNA molecules elicit the sequence-specific degradation of a complementary mRNA target. Previous studies have reported the effects of siRNA in animal models of AR (16-22). These previous studies 
targeted CD40, signal transducer and activator of transcription 6, neurokinin-1 receptor, C-C chemokine receptor type 3, transient receptor potential cation subfamily $\mathrm{M}$ member 8 , phosphatidylinositol-3-kinase $\mathrm{C} 2 \beta$ and calcium release-activated calcium channel protein 1 . In the present study, the effect of the siRNA-mediated silencing of CD86 in DCs on Treg and Th2 cell cytokine production was examined in vitro.

\section{Materials and methods}

Clinical specimens. Patients with AR $(n=39)$ and healthy control subjects $(n=36)$ participated in the present study. The patients were diagnosed based on the criteria of the Initiative on Allergic Rhinitis and its Impact on Asthma (23), including a characteristic history of paroxysmal sneezing, watery rhinorrhea, nasal obstruction, positive specific immunoglobulin E (IgE), a positive skin prick test (SPT) and nasal examination. All patients were prohibited from taking any medication within 4 weeks of the study. Patients with infectious rhinitis, an occupational or drug-induced etiology, and those with any complications, were excluded. Healthy control subjects were selected based on the following criteria: No history of allergic diseases, a negative SPT or specific IgE to common allergens (total IgE levels $<100 \mathrm{kU} /$ liter). In the siRNA group, DCs from patients with AR were treated with siRNA intervention. In the non-siRNA group, DCs from patients with AR were not treated with siRNA intervention. The healthy control group consisted of healthy subjects. The present study was approved by the Ethics Committee of Chongqing Medical University. Informed consent for all subjects was obtained from parents or legal guardians. All procedures strictly conformed to the principles outlined in the Declaration of Helsinki.

Generation of DCs. A total of $10 \mathrm{ml}$ blood was collected from each subject. Peripheral blood mononuclear cells (PBMCs) were isolated by means of Ficoll-Plaque Plus density gradient centrifugation at $800 \times \mathrm{g}$ for $30 \mathrm{~min}$ at $4^{\circ} \mathrm{C}$. After trypan blue staining, the viability $(>95 \%)$ of PBMCs was detected by an exclusion assay under an optical microscope. CD14 ${ }^{+}$ monocytes were isolated using magnetic separation (Miltenyi Biotec $\mathrm{GmbH})$. Isolated $\mathrm{CD} 14^{+}$cells were transferred into 96-well plates, at a density of $1.0 \times 10^{6}$ cells/well in $0.1 \mathrm{ml}$ of complete medium, for 6 days to promote differentiation into immature DCs (imDCs). The complete medium consisted of RPMI-1640 (R\&D Systems Inc.), 10\% fetal calf serum (R\&D Systems Inc.), $100 \mathrm{U} / \mathrm{ml}$ penicillin, $100 \mathrm{ng} / \mathrm{ml}$ streptomycin, $100 \mathrm{ng} / \mathrm{ml}$ recombinant human granulocyte-macrophage colony-stimulating factor (PeproTech, Inc.) and $50 \mathrm{ng} / \mathrm{ml}$ recombinant human IL-4 (PeproTech, Inc.). Half the volume of the old media was removed and fresh media was added every $48 \mathrm{~h}$. After 6 days of culture, $100 \mathrm{ng} / \mathrm{ml}$ lipopolysaccharide (LPS; Sigma-Aldrich; Merck KGaA) was added to the cells for $24 \mathrm{~h}$. The mDCs were obtained on day 7.

Transfection of DCs with CD86 siRNA in a lentiviral vector. CD86 siRNA in a lentiviral vector was purchased from Shanghai GenePharma Co., Ltd. The CD86 siRNA sequence was AGACCACATTCCTTGGATT. The component sequence of the lentivirus vector was human U6, multiple cloning sites, cytomegalovirus, green fluorescent protein (GFP), simian virus 40 and neomycin. The expression of GFP indicated a successful transfection. Preliminary experiments to calculate the multiplicity of infection (MOI) were carried out; an MOI of 20 corresponded to a transfection efficiency of $60.2 \%$, while an MOI of 10 corresponded to a transfection efficiency of $43.5 \%$. Transfections were performed when DCs reached a confluency of $30-50 \%$, according to the manufacturer's protocol. imDCs were seeded in 96-well plates and were transfected with CD86 siRNA in a lentiviral vector at an MOI of 20 on day 3 of the $\mathrm{CD} 14^{+}$cell culture. After $72 \mathrm{~h}, 100 \mathrm{ng} / \mathrm{ml} \mathrm{LPS}$ was added to the DCs for $24 \mathrm{~h}$. To exclude the effect of transfection reagent on CD86 expression, a preliminary experiment was carried out (data not shown). CD86 expression was determined by flow cytometry through PE-conjugated anti-human CD86 mAb as described below. The results showed that transfection with an empty lentiviral vector (Shanghai GenePharma Co., Ltd.) had no effect on CD86 expression (data not shown). To determine if siRNA had been successfully transfected into DCs, the expression of GFP was observed using fluorescence microscopy at $6 \mathrm{~h}$ post transfection. The transfection efficiency was determined by flow cytometry at $48 \mathrm{~h}$ after transfection.

Flow cytometry. DCs were stained with phycoerythrin (PE)-Cyanine5-conjugated anti-human leukocyte antigen-DR isotype (HLA-DR) mouse antibody (mAb) (eBioscience; Thermo Fisher Scientific, Inc.), PE-conjugated anti-human CD86 mAb (eBioscience; Thermo Fisher Scientific, Inc.) and FITC-conjugated anti-human CD80 mAb (eBioscience; Thermo Fisher Scientific, Inc.). A minimum of 20,000 events were collected in each analysis. DCs were analyzed using a FACScan flow cytometer (BD Biosciences). Further data analysis was performed using CellQuest software (CellQuest Pro 5.2.1, BD Biosciences).

Co-culture with $C D 4^{+} T$ cells. Peripheral $\mathrm{CD}^{+} \mathrm{T}$ cells were isolated from PBMCs using human CD4 microbeads (Miltenyi Biotec $\mathrm{GmbH}$ ). Co-culture was performed with a $\mathrm{DC} / \mathrm{T}$ cell ratio of 1:4 in 24-well plates for 7 days without any stimulating factors. The culture plate was incubated at $37^{\circ} \mathrm{C}$ in a humidified incubator with $5 \% \mathrm{CO}_{2}$. The supernatant and co-cultured cells were collected for the following assays. The supernatant was collected and stored at $-80^{\circ} \mathrm{C}$ until cytokine levels were measured.

ELISA for IL-4, IL-5 and TGF- $\beta 1$. The levels of IL-4 (cat. no. BMS225HS), IL-5 (cat. no. BMS278INST) and TGF- $\beta 1$ (cat. nos. BMS249/4BMS249/4TEN) in the supernatant was detected using ELISA (eBioscience; Thermo Fisher Scientific, Inc.), according to the manufacturer's instructions. The limits of detection for Il-4, Il-5 and TGF- $\beta 1$ were 0.1 , 10 and $8.6 \mathrm{pg} / \mathrm{ml}$, respectively.

Reverse transcription-quantitative $(R T-q) P C R$ analysis of forkhead box P3 (FOXP3) and GATA-binding protein 3 (GATA-3) expression. Total RNA was isolated using a Total RNA Extraction kit (BioTeke Corporation). RT was performed using a Prime Script RT Reagent kit (Takara, Biotechnology Co., Ltd.), according to the manufacturer's instructions. The sequences of the primers (Sangon Biotech, Shanghai, China) were as follows: FOXP3 forward, 5'-AGGGACCAAGAA 

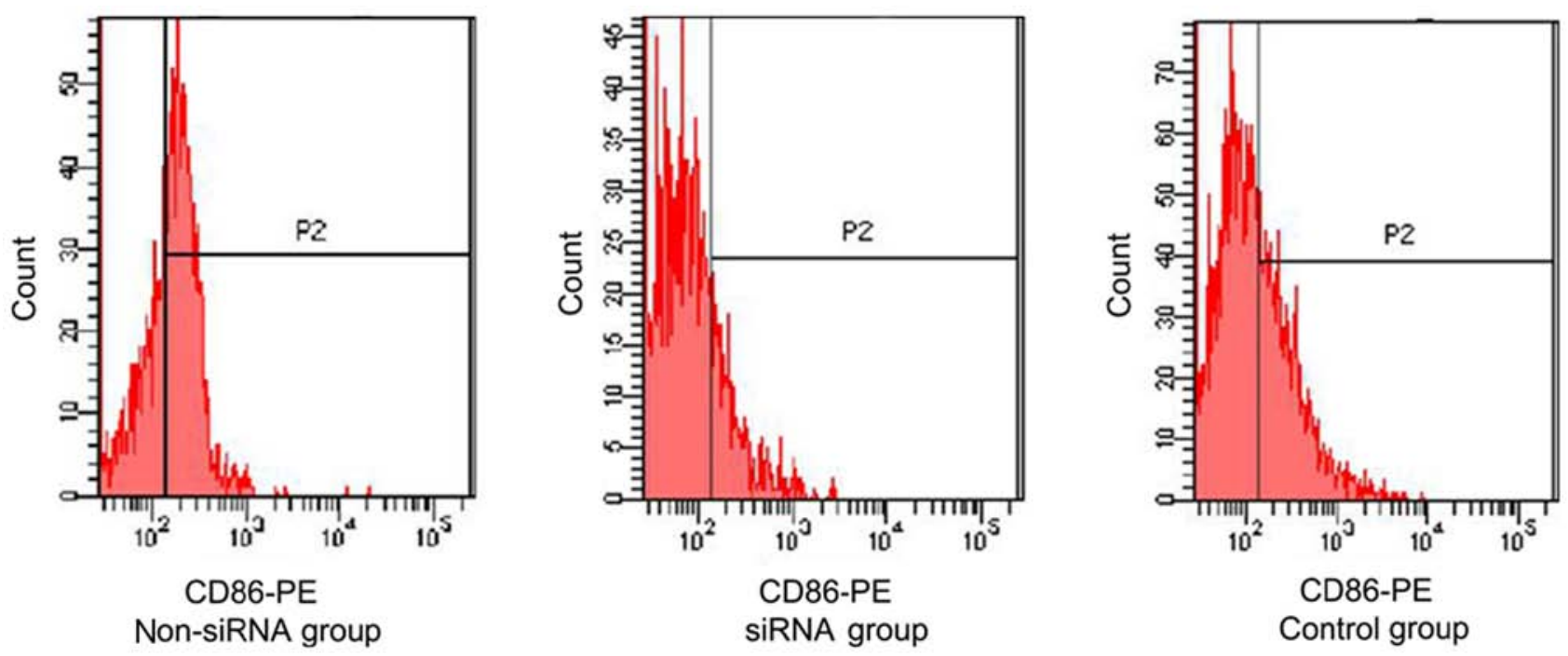

Figure 1. Expression of CD86 on dendritic cells was determined by flow cytometry. The expression levels of CD86 in the non-siRNA, siRNA and the normal control groups were $61.2 \pm 15.46,17.2 \pm 3.17$ and $37.6 \pm 5.93$, respectively. The CD86 expression levels in the siRNA group decreased significantly compared with those in the non-siRNA and the healthy control groups $(\mathrm{P}<0.05)$. siRNA, small interfering RNA; CD86, T-lymphocyte activation antigen CD86; PE, phycoerythrin.

GTGAGGTTTC-3' and reverse, 5'-TGGGGTTTGTGTTGA GTGAG-3'; GATA-3 forward, 5'-AGACCACCACAACCA CACTCT-3' and reverse, 5'-GATGCCTTCCTTCTTCAT AGTCA-3'; $\beta$-actin forward, 5'-AGCGAGCATCCCCCAAAG TT-3' and reverse, 5'-GGGCACGAAGGCTCATCATT-3'. The mRNA levels of FOXP3 and GATA-3 were quantified using qPCR as described previously (5). A brief introduction is as follows. Reactions were heated to $95^{\circ} \mathrm{C}$ for 4 min followed by 30 cycles of denaturation at $95^{\circ} \mathrm{C}$ for $45 \mathrm{sec}$, annealing at $55^{\circ} \mathrm{C}$ (FOXP3), $58^{\circ} \mathrm{C}$ (GATA-3), $59^{\circ} \mathrm{C}$ ( $\beta$-actin) for $5 \mathrm{sec}$, and extension at $72^{\circ} \mathrm{C}$ for $10 \mathrm{~min}$. All PCR reactions were performed in duplicate. To confirm the specificity of the PCR reaction, PCR products were analyzed with $3 \%$ agarose gel electrophoresis and ethidium bromide staining, followed by visualization with anultraviolet rays transilluminator.

Western blot analysis of FOXP3 and GATA3 expression. Proteins were extracted using RIPA (Thermo Fisher Scientific, Inc.) from control co-cultured cells or following transfection with empty vector or CD86-siRNA containing vector. The protein levels of FOXP3 and GATA-3 were determined by western blot analysis as described previously (5). The dilution of FOXP3 monoclonal antibody (cat. no. 14-7979-82, eBioscience; Thermo Fisher Scientific, Inc.) was 1:1,000. The dilution of GATA3 Monoclonal Antibody (cat. no. 66400-1-IG, eBioscience; Thermo Fisher Scientific, Inc.) was 1:2,000. The dilution of Goat anti-Mouse IgG (H+L) Cross-Adsorbed Secondary Antibody (cat. no. G-21040, Invitrogen; Thermo Fisher Scientific, Inc.) was 1:2,000. The incubation temperature of the primary antibody was $4^{\circ} \mathrm{C}$ and the incubation duration was overnight. The incubation temperature of the secondary antibody was room temperature and the incubation duration was $1 \mathrm{~h}$. Data were analyzed with Quantity One software, version 4.52 (Bio-Rad Laboratories, Inc.).

Statistical analysis. Each experiment was performed a minimum of five times. All results are presented as the mean \pm SD. Data were analyzed by one-way ANOVA followed by Bonferroni post-hoc test using SPSS v.11.5 software (SPSS, Inc.). $\mathrm{P}<0.05$ was considered to indicate a statistically significant difference.

\section{Results}

Expression of $m D C$ surface molecules. The expression of CD80, CD86 and HLA-DR on mDCs was analyzed using flow cytometry. There were no significant differences in the expression of CD80 and HLA-DR on mDCs between the AR and the healthy control groups (data not shown). However, mDCs from the AR group exhibited a significantly higher expression level of CD86 compared with the control group ( $\mathrm{P}<0.05$; Fig. 1$)$. CD83 is an important surface antigen expressed by mDCs. To verify that $\mathrm{mDCs}$ had been induced, CD83 expression levels on mDCs were analyzed by flow cytometry in a preliminary experiment. The mDCs were found to exhibit a significantly higher CD83 expression level compared with the imDCs (data not shown).

Effects of siRNA on surface molecule expression in DCs. At $6 \mathrm{~h}$ after CD86 siRNA transfection, the efficiency of transfection of the DCs was assessed by fluorescence microscopy and the transfection efficiency was determined by flow cytometry. DCs were successfully transfected with CD86 siRNA using a lentiviral vector. As shown in Fig. 2, the transfection efficiency of CD86-siRNA was $60.2 \%$, as determined by assessing the number of GFP-positive cells. At $96 \mathrm{~h}$ after transfection, the expression of CD86 on DCs was determined by flow cytometry. CD86 expression levels were significantly lower in the siRNA group (17.2 \pm 3.17$)$ compared with those in the non-siRNA (61.2 \pm 15.46$)$ and control (37.6 \pm 5.93$)$ groups. The difference was statistically significant $(\mathrm{P}<0.05$; Fig. 1$)$. However, the CD80 expression levels in the non-siRNA, siRNA and control groups were $85.45 \pm 17.81,83.79 \pm 14.27$ and $86.36 \pm 18.96 \%$, respectively; the differences were not significant $(\mathrm{P}>0.05$; data not shown). 

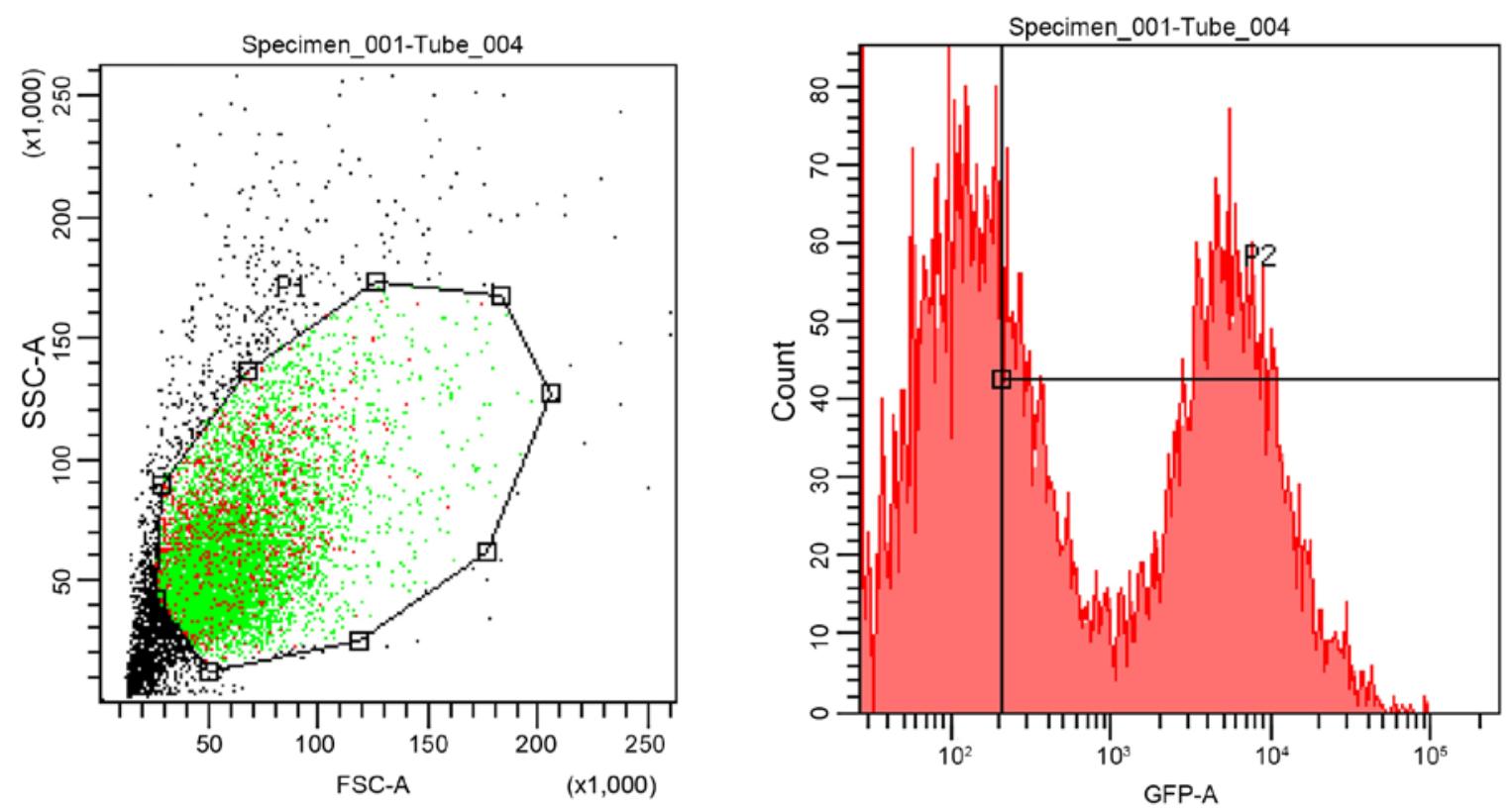

Figure 2. Transfection efficiency was determined by flow cytometry. A multiplicity of infection of 20 corresponded to a transfection efficiency of $60.2 \%$. SSC, side scatter; FSC, forward scatter; GFP, green fluorescent protein.
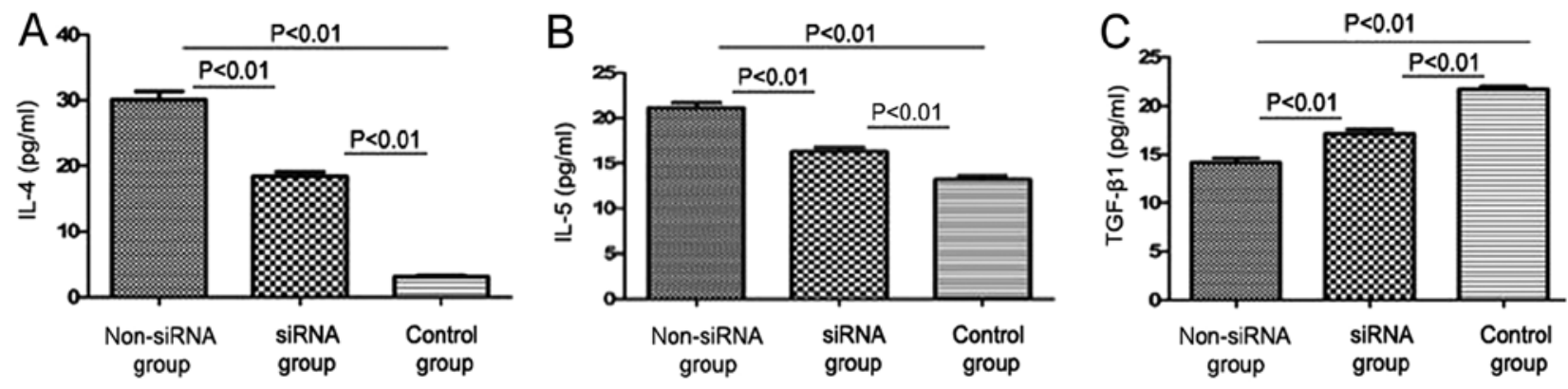

Figure 3. Levels of IL-4, IL-5 and TGF- $\beta 1$ in the supernatant of DC:CD4 ${ }^{+}$T cell co-culture. The levels of (A) IL-4 and (B) IL-5 and (C) TGF- $\beta 1$ were determined using ELISA. IL-4 and IL-5 levels were significantly lower, but the level of TGF- $\beta 1$ was significantly higher after T-lymphocyte activation antigen CD86 knockdown in patients with AR compared with the non-siRNA AR group. Data are presented as the mean \pm SD. IL, interleukin; TGF- $\beta 1$, transforming growth factor- $\beta 1$; DCs, dendritic cells; AR, allergic rhinitis; siRNA, small interfering RNA.

$I L-4, I L-5$ and TGF- $\beta 1$ secretion by $T$ cells co-cultured with $D C s$. The expression levels of IL-4 and IL-5 were significantly lower in the supernatant of the co-cultured cells in the siRNA-treated group $(19.07 \pm 0.58$ and $15.9 \pm 0.51 \mathrm{pg} / \mathrm{ml}$, respectively) compared with those in the non-siRNA group (30.23 \pm 1.37 and $22.04 \pm 0.78 \mathrm{pg} / \mathrm{ml}$, respectively); the differences were statistically significant $(\mathrm{P}<0.01$; Fig. $3 \mathrm{~A}$ and $\mathrm{B})$. Moreover, IL-4 and IL-5 expression levels were significantly higher in the non-siRNA and siRNA groups compared with those in the control group ( $\mathrm{P}<0.01$; Fig. $3 \mathrm{~A}$ and $\mathrm{B})$. The expression of TGF- $\beta 1$ in the supernatant of the co-cultured cells was significantly higher following transfection with siRNA targeting CD86 $(16.22 \pm 0.48 \mathrm{pg} / \mathrm{ml})$ compared with that in the non-siRNA group $(14.27 \pm 0.51 \mathrm{pg} / \mathrm{ml})$. The difference was statistically significant $(\mathrm{P}<0.01$; Fig. $3 \mathrm{C})$.

FOXP 3 and GATA 3 mRNA expression by T cells co-cultured with DCs. The expression level of FOXP3 mRNA was higher in the siRNA-treated group $(0.541 \pm 0.027)$ compared with that in the non-siRNA group $(0.412 \pm 0.035$; $\mathrm{P}<0.01$; Fig. 4$)$, whereas GATA3 mRNA expression was significantly lower in the siRNA group $(0.196 \pm 0.013)$ compared with that in the non-siRNA group $(0.305 \pm 0.029)$. The difference was statistically significant $(\mathrm{P}<0.01$; Fig. 4).

FOXP3 and GATA3 protein expression by T cells co-cultured with DCs. The expression level of the FOXP3 protein was considerably higher in the siRNA group $(0.619 \pm 0.035)$ compared with that in the non-siRNA group $(0.217 \pm 0.021$; $\mathrm{P}<0.01$; Fig. 5). However, the GATA3 protein expression level in the siRNA group $(0.317 \pm 0.027)$ was significantly lower compared with that in the non-siRNA group $(0.917 \pm 0.046$; $\mathrm{P}<0.01$; Fig. 5). These results were consistent with those of the RT-qPCR analysis.

\section{Discussion}

In the present study, the mDCs of patients with AR were found to express high levels of the co-stimulatory molecule CD86. Following transfection with CD86 siRNA using a lentiviral 


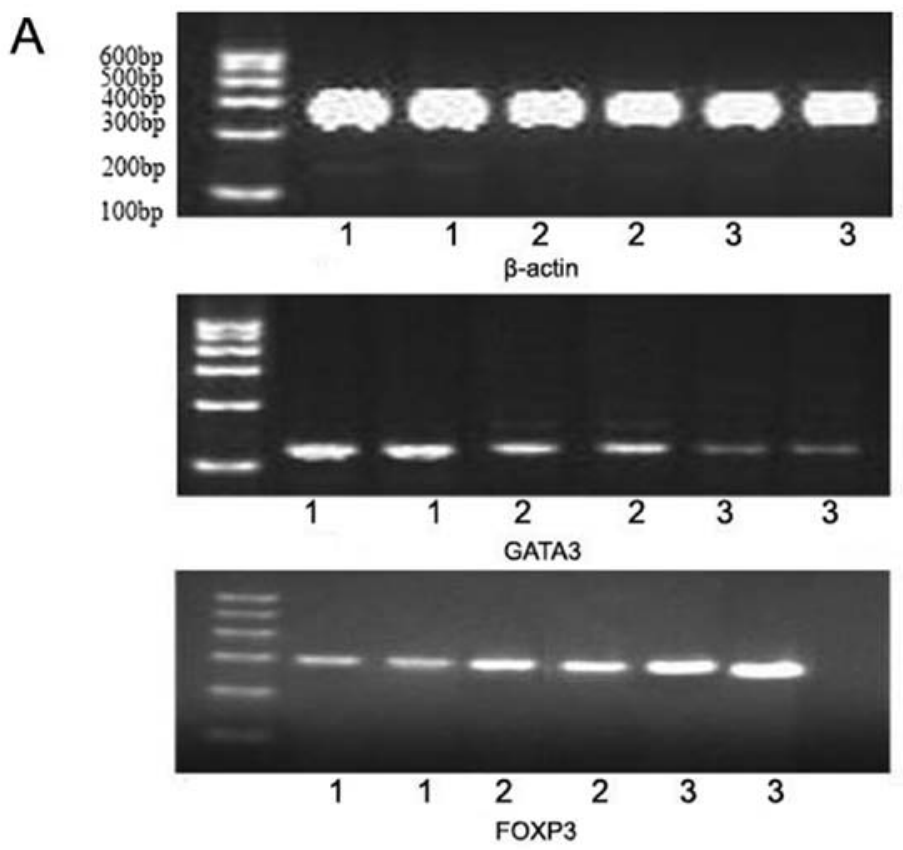

B

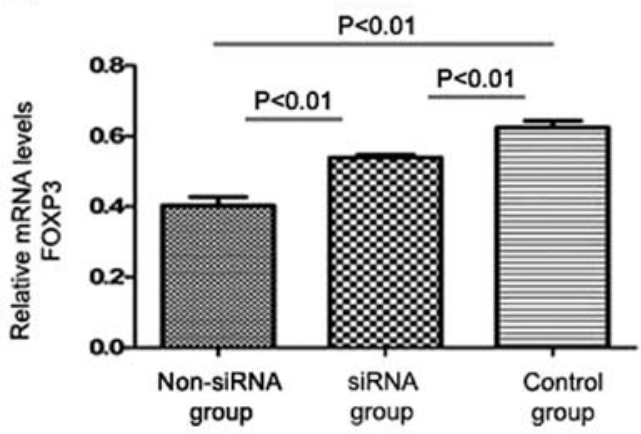

C

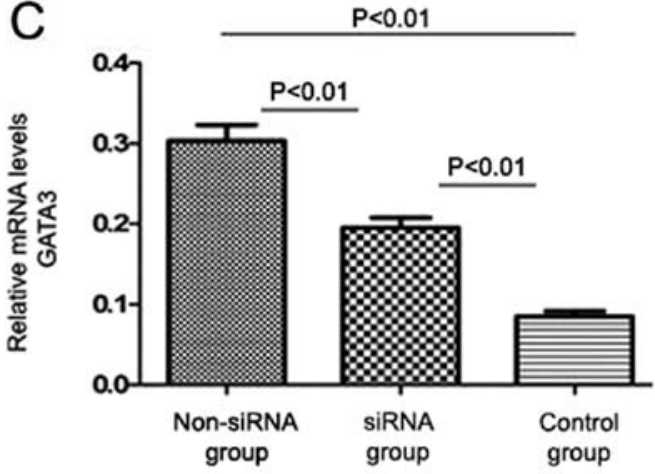

Figure 4. Expression of FOXP3 and GATA3 mRNA in dendritic cell/CD4 ${ }^{+} \mathrm{T}$ cell co-culture. (A) Expression levels of FOXP3 and GATA3 mRNA were determined using reverse transcription-quantitative PCR. (B) The expression of FOXP3 mRNA was higher in the siRNA-treated group compared with that in the non-siRNA treated group. (C) GATA3 mRNA expression was lower in the siRNA group compared with that in the non-siRNA treated group. Lane 1, non-siRNA group; 2, siRNA group; 3, control group. FOXP3, forkhead box P3; GATA3, GATA-binding protein 3.

vector, the expression levels of CD86 on DCs were markedly reduced. CD86 siRNA-transfected DCs induced Th2 cell deficiency and Treg cell hyperactivity. These results indicated that CD86 plays a potentially important role in the regulation of the Treg/Th2 cell imbalance in allergic inflammation.

The co-stimulatory molecule CD86 is prominent in allergic inflammation, including asthma and atopic dermatitis (24-26). Despite sharing the same T cell stimulatory receptor, CD86 appears to induce more potent allergic responses compared with CD80. Wong et al (24) reported that steroid- and non-steroid-treated asthmatic patients expressed higher levels of plasma CD86, but not CD80, compared with control subjects. The expression of CD86 on airway DCs was increased gradually following continuous stimulation by allergens (11). In another animal model of asthma, a continuous allergen challenge induced the maturation of lung DCs and an increased expression of CD86 (12). It was demonstrated that $\mathrm{T}$ cell activation is closely associated with increased levels of CD86, but not CD80, on the surface of DCs during late-phase airway allergic response using siRNA targeting CD86 (25). It has been shown that the local application of CD86 siRNA targeting cutaneous DCs improved allergic skin inflammation (26). In the present study, mDCs from the peripheral blood of patients with AR were found to express higher levels of CD86, but not CD80, compared with the healthy control group. A similar observation in DCs was described by KleinJan et al (15). DCs in the nasal mucosa of symptomatic AR patients expressed higher levels of CD86, which was associated with Th2-driven allergic responses. Together with the study by KleinJan et al (15), the results of the present study suggested that mDCs express high levels of CD86, which may play an active role in allergic inflammation. Therefore, CD86 molecules on DCs may represent a novel therapeutic target for AR.

The role of the co-stimulatory molecule CD86 in the differentiation and activation of $\mathrm{T}$ cells was also investigated in the present study. The data in the present study demonstrated that $\mathrm{T}$ cells co-cultured with DCs produced higher levels of TGF- $\beta 1$ and lower levels of IL- 4 and IL-5 in the siRNA group compared with those in the non-siRNA and healthy control group. The expression of GATA-3 was lower and that of FOXP3 higher at the mRNA and protein levels in the siRNA-treated group compared with in the non-siRNA and healthy control group. These results indicated that 

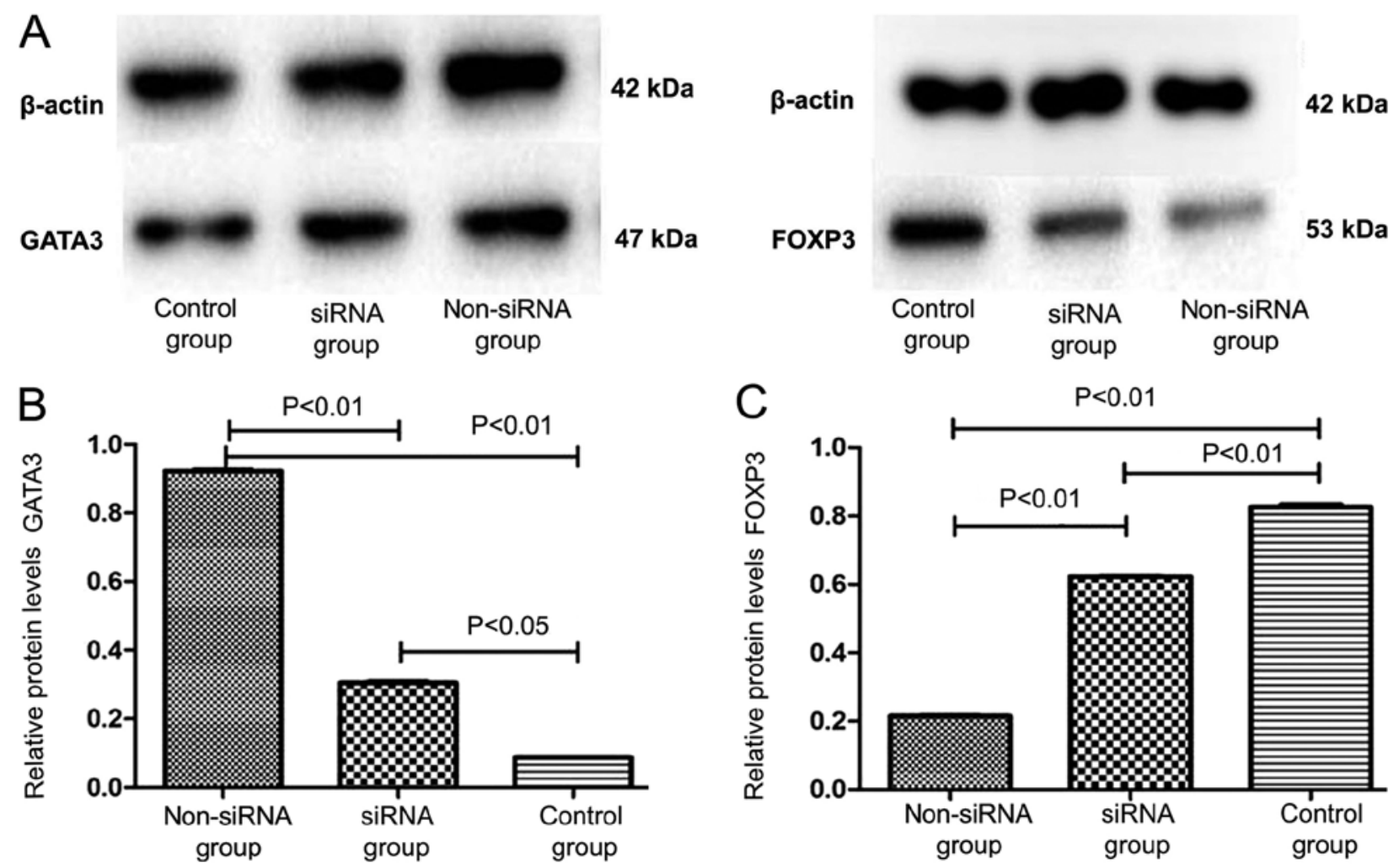

Figure 5. Expression of FOXP3 and GATA3 protein in dendritic cell/CD4+ T co-culture. T cell co-culture was investigated by western blot analysis. (A) Western blot analysis for GATA3 and FOXP3 protein. (B) GATA3 protein expression was lower in the siRNA group compared with that in the non-siRNA group. (C) FOXP3 protein expression was higher in the siRNA group compared with that in the non-siRNA group. FOXP3, forkhead box P3; GATA3, GATA-binding protein 3; siRNA, small interfering RNA.

CD86-silenced DCs may regulate the Treg/Th2 cell imbalance in allergic inflammation. There are two aspects to the effect of CD86-silenced DCs on T cells: Th2 deficiency and Treg hyperactivity. CD86-silenced DCs decrease the number of IL-4- and IL-5-producing Th2 cells. The presence of CD86 on DCs is required for Th2 cell differentiation and activation (27). Previous studies using animal models of asthma have demonstrated that anti-CD86 mAb treatment, but not anti-CD80 mAb treatment, effectively inhibited antigen-specific IgE and Th2 cytokine production (27-29). In addition, the blockade of CD86 may suppress airway hyper-responsiveness and Th2-driven allergic responses in allergic mice (30). The present study also demonstrated that CD86-silenced DCs did not promote $\mathrm{T}$ cell activation or production of IL-4 and IL-5, whereas CD86-silenced DCs increased the number of TGF- $\beta 1$-producing Treg cells. CD86 plays a crucial role in DC maturation (5). Immature or semi-mature DCs induce immune tolerance $(31,32)$. The $\mathrm{mAb}$ against CD3 on DCs increased the number of antigen-specific Tregs in autoimmune diabetes (33). Knockdown of CD40 using siRNA in OVA-exposed DCs induced the generation of allergen-specific Treg cells, which suppressed allergic responses in vivo (16). The present study demonstrated that the expression of FOXP3 at the mRNA or protein level was higher in the CD86 siRNA-treated group compared with that in the control group. These results suggested that the effects of CD86-silenced DCs on allergic inflammation are closely associated with the regulation of the Treg/Th2 cell imbalance. Using CD86 siRNA-treated DCs to skew the immune response from Th2 cells towards Treg cells may be a viable therapeutic approach to the treatment of AR.

To the best of our knowledge, the present study is the first to introduce an effective method for inhibiting the upregulation of CD86 on DCs from patents with AR using siRNA. An advantage of siRNA is the ability to specifically knock down a designated gene without directly affecting the expression of any other mRNAs. CD86 expression on DCs decreased significantly following siRNA treatment, whereas HLA-DR and CD80 expression exhibited little or no change.

The present study had some limitations. For example, Treg hyperactivity does not necessarily mean an increase in function, it may also be due to an increase in the quantity of Treg cells. The increased expression of FOXP3 at the mRNA and protein level does not indicate amplification of the Treg function or population; changes in function need to be verified using mixed lymphocyte reaction experiments. Additionally, the data provided in the present study are from in vitro experiments and no disease model was used. These points will be addressed in future studies.

In conclusion, the findings of the present study suggested that mDCs from patients with AR express high levels of the co-stimulatory molecule CD86, but not of CD80. CD86 expression in DCs was specifically reduced using a lentiviral vector expressing siRNA. CD86 siRNA-treated DCs altered the Treg/Th2 cell balance. The findings from the present study indicated that CD86 may play an important role in the pathogenesis of allergic inflammation and may be a novel target for RNA interference that could be used in the treatment of AR. 


\section{Acknowledgements}

Not applicable.

\section{Funding}

The present study was supported by the National Natural Science Foundation Project (grant no. 81500776) and the Fundamental and Advanced Research Program of Chongqing (grant no. cstc2015jcyjA10103).

\section{Availability of materials and data}

The datasets used and/or analyzed during the current study are available from the corresponding author on reasonable request.

\section{Authors' contributions}

RS made substantial contributions to the experimental design, generation and transfection of DCs, co-culture of cells, acquisition of data and analysis of data. YY and ZG were responsible for the collection of clinical specimens and the ELISA to determine the expression levels of cytokines. CZ and XT were responsible for flow cytometry. WK and PW carried out RT-qPCR and western blotting analysis of FOXP3 and GATA3 expression levels. In addition, XT was also involved in drafting the manuscript and revising it critically for important intellectual content. All the authors have read and approved the final version of this manuscript.

\section{Ethics approval and consent to participate}

The present study was approved by the Ethics Committee of Chongqing Medical University. Informed consent was obtained from the parents or legal guardians of all subjects. All procedures strictly conformed to the principles outlined in the Declaration of Helsinki.

\section{Patient consent for publication}

Not applicable.

\section{Competing interests}

The authors declare that they have no competing interests.

\section{References}

1. Pilette C, Jacobson MR, Ratajczak C, Detry B, Banfield G, VanSnick J, Durham SR and Nouri-Aria KT: Aberrant dendritic cell function conditions Th2-cell polarization in allergic rhinitis. Allergy 68: 312-321, 2013.

2. Skrindo I, Ballke C, Gran E, Johansen FE, Baekkevold ES and Jahnsen FL: IL-5 production by resident mucosal allergen-specific $\mathrm{T}$ cells in an explant model of allergic rhinitis Clin Exp Allergy 45: 1296-1304, 2015.

3. Akdis M, Verhagen J, Taylor A, Karamloo F, Karagiannidis C, Crameri R, Thunberg S, Deniz G, Valenta R, Fiebig H, et al Immune responses in healthy and allergic individuals are characterized by a fine balance between allergen-specific $\mathrm{T}$ regulatory 1 and T helper 2 cells. J Exp Med 199: 1567-1575, 2004 .
4. Lee JH, Yu HH, Wang LC, Yang YH, Lin YT and Chiang BL: The levels of CD4+CD25+ regulatory $T$ cells in paediatric patients with allergic rhinitis and bronchial asthma. Clin Exp Immunol 148: 53-63, 2007.

5. Sun R, Tang XY and Yang Y: Immune imbalance of regulatory T/type 2 helper cells in the pathogenesis of allergic rhinitis in children. J Laryngol Otol 130: 89-94, 2016.

6. Mellman I and Steinman RM: Dendritic cells: Specialized and regulated antigen processing machines. Cell 106: 255-258, 2001.

7. Lambrecht BN and Hammad H: The role of dendritic and epithelial cells as master regulators of allergic airway inflammation. Lancet 376: 835-843, 2010.

8. Lim TS, Goh JK, Mortellaro A, Lim CT, Hämmerling GJ and Ricciardi-Castagnoli P: CD80 and CD86 differentially regulate mechanical interactions of T-cells with antigen-presenting dendritic cells and B-cells. PLoS One 7: e45185, 2012.

9. Kuchroo VK, Das MP, Brown JA, Ranger AM, Zamvil SS, Sobel RA, Weiner HL, Nabavi N and Glimcher LH: B7-1 and B7-2 costimulatory molecules activate differentially the Th1/Th2 developmental pathways: Application to autoimmune disease therapy. Cell 80: 707-718, 1995.

10. Lenschow DJ, Ho SC, Sattar H, Rhee L, Gray G, Nabavi N, Herold KC and Bluestone JA: Differential effects of anti-B7-1 and anti-B7-2 monoclonal antibody treatment on the development of diabetes in the nonobese diabetic mouse. J Exp Med 181: $1145-1155,1995$.

11. Vermaelen $\mathrm{K}$ and Pauwels R: Accelerated airway dendritic cell maturation, trafficking, and elimination in a mouse model of asthma. Am J Respir Cell Mol Biol 29: 405-409, 2003.

12. Gajewska BU, Swirski FK, Alvarez D, Ritz SA, Goncharova S, Cundall M,Snider DP, Coyle AJ, Gutierrez-Ramos JC,Stämpfli MR and Jordana M: Temporal-spatial analysis of the immune response in a murine model of ovalbumin-induced airways inflammation. Am J Respir Cell Mol Biol 25: 326-334, 2001.

13. Till SJ, Jacobson MR, O'Brien F, Durham SR, KleinJan A, Fokkens WJ, Juliusson S and Löwhagen O: Recruitment of CD1a+ Langerhans cells to the nasal mucosa in seasonal allergic rhinitis and effects of topical corticosteroid therapy. Allergy 56: 126-131, 2001.

14. Holm A, Dijkstra M, Kleinjan A, Severijnen LA, Boks S, Mulder $\mathrm{P}$ and Fokkens W: Fluticasone propionate aqueous nasal spray reduces inflammatory cells in unchallenged allergic nasal mucosa: Effects of single allergen challenge. J Allergy Clin Immunol 107: 627-633, 2001.

15. KleinJan A, Willart M, van Rijt LS, Braunstahl GJ, Leman K, Jung S, Hoogsteden HC and Lambrecht BN: An essential role for dendritic cells in human and experimental allergic rhinitis. J Allergy Clin Immunol 118: 1117-1125, 2006.

16. Suzuki M, Zheng X, Zhang X, Zhang ZX, Ichim TE, Sun H, Nakamura Y, Inagaki A, Beduhn M, Shunnar A, et al: A novel allergen-specific therapy for allergy using CD40-silenced dendritic cells. J Allergy Clin Immunol 125: 737, 2010.

17. Hosoya K, Satoh T, Yamamoto Y, Saeki K, Igawa K, Okano M, Moriya T, Imamura O, Nemoto Y and Yokozeki H: Gene silencing of STAT6 with siRNA ameliorates contact hypersensitivity and allergic rhinitis. Allergy 66: 124-131, 2011.

18. Wang $\mathrm{H}$, Zhang $\mathrm{R}$, Wu J and $\mathrm{Hu} \mathrm{H}$ : Knockdown of neurokinin-1 receptor expression by small interfering RNA prevents the development of allergic rhinitis in rats. Inflamm Res 62: 903-910, 2013.

19. Zhu XH, Liao B, Liu K and Liu YH: Effect of RNA interference therapy on the mice eosinophils CCR3 gene and granule protein in the murine model of allergic rhinitis. Asian Pac J Trop Med 7: 226-230, 2014.

20. Cho Y, Jang Y, Yang YD, Lee CH, Lee Y and Oh U: TRPM8 mediates cold and menthol allergies associated with mast cell activation. Cell Calcium 48: 202-208, 2010.

21. Srivastava S, Cai X, Li Z, Sun Y and Skolnik EY: Phosphatidylinositol-3-kinase C2 $\beta$ and TRIM27 function to positively and negatively regulate IgE receptor activation of mast cells. Mol Cell Biol 32: 3132-3139, 2012.

22. Wang Y, Lin L and Zheng C: Downregulation of Orail expression in the airway alleviates murine allergic rhinitis. Exp Mol Med 44: 177-190, 2012.

23. Bousquet J, Khaltaev N, Cruz AA, Denburg J, Fokkens WJ Togias A, Zuberbier T, Baena-Cagnani CE, Canonica GW, van Weel $\mathrm{C}$, et al: Allergic rhinitis and its impact on asthma (ARIA) 2008 update (in collaboration with the World Health Organization, GA(2)LEN and AllerGen). Allergy 63 (Suppl 86) S8-S160, 2008. 
24. Wong CK, Lun SW, Ko FW, Ip WK, Hui DS and Lam CW: Increased expression of plasma and cell surface co-stimulatory molecules CTLA-4, CD28 and CD86 in adult patients with allergic asthma. Clin Exp Immunol 141: 122-129, 2005.

25. Asai-Tajiri Y, Matsumoto K, Fukuyama S, Kan-O K, Nakano T, Tonai K, Ohno T, Azuma M, Inoue H and Nakanishi Y: Small interfering RNA against CD86 during allergen challenge blocks experimental allergic asthma. Respir Res 15: 132, 2014.

26. Ritprajak P, Hashiguchi M and Azuma M: Topical application of cream-emulsified CD86 siRNA ameliorates allergic skin disease by targeting cutaneous dendritic cells. Mol Ther 16: 1323-1330, 2008.

27. Nakajima A, Watanabe N, Yoshino S, Yagita H, Okumura K and Azuma M: Requirement of CD28-CD86 co-stimulation in the interaction between antigen-primed T helper type 2 and $\mathrm{B}$ cells. Int Immunol 9: 637-644, 1997.

28. Tsuyuki S, Tsuyuki J, Einsle K, Kopf $M$ and Coyle AJ: Costimulation through B7-2 (CD86) is required for the induction of a lung mucosal Thelper cell 2 (TH2) immune response and altered airway responsiveness. J Exp Med 185: 1671-1679, 1997.

29. Huh JC, Strickland DH, Jahnsen FL, Turner DJ, Thomas JA, Napoli S, Tobagus I, Stumbles PA, Sly PD and Holt PG: Bidirectional interactions between antigen-bearing respiratory tract dendritic cells (DCs) and T cells precede the late phase reaction in experimental asthma: DC activation occurs in the airway mucosa but not in the lung parenchyma. J Exp Med 198: 19-30, 2003.
30. Crosby JR, Guha M, Tung D, Miller DA, Bender B, Condon TP, York-DeFalco C, Geary RS, Monia BP, Karras JG and Gregory SA: Inhaled CD86 antisense oligonucleotide suppresses pulmonary inflammation and airway hyper-responsiveness in allergic mice. J Pharmacol Exp Ther 321: 938-946, 2007.

31. Banchereau J and Steinman RM: Dendritic cells and the control of immunity. Nature 392: 245-252, 1998.

32. Lutz MB and Schuler G: Immature, semi-mature and fully mature dendritic cells: Which signals induce tolerance or immunity? Trends Immunol 23: 445-449, 2002.

33. You S, Leforban B, Garcia C, Bach JF, Bluestone JA and Chatenoud L: Adaptive TGF-beta-dependent regulatory T cells control autoimmune diabetes and are a privileged target of anti-CD3 antibody treatment. Proc Natl Acad Sci USA 104: 6335-6340, 2007. 